\title{
Effect of dietary cassava peel meal supplemented with methionine and multienzyme on hemo-biochemical indices, digestibility, and antioxidants in rabbits
}

\author{
Olugbenga D. Oloruntola ${ }^{1,2}$
}

\begin{abstract}
Background: The effects of cassava peel meal (CPM) supplemented with methionine (MET) and multienzyme (ENZ) was studied using 2405 -week-old rabbits in a completely randomized design arranged in $2 \times 2 \times 2$ factorial ( 2 CPM levels, 0 and $350 \mathrm{~g} / \mathrm{kg} ; 2$ methionine levels, 5.6 and $8.3 \mathrm{~g} / \mathrm{kg}$; and 2 enzyme levels, 0 and $0.5 \mathrm{~g} / \mathrm{kg}$ ).

Results: The CPM inclusion reduced $(P<0.05)$ leucine, lysine, valine, and threonine digestibility, while the digestibility of leucine, lysine, and valine increased $(P<0.05)$ by high methionine level. Multienzyme increased leucine, lysine, and valine digestibility. CPM reduced $(P<0.01)$ red blood cell $(\mathrm{RBC})$ count and the total serum protein. Higher methionine supplementation increased the serum total protein by $9.2 \%$. The CPM inclusion increased bilirubin, alanine aminotransferase (ALT), and aspartate aminotransferase (AST). Methionine and enzyme supplementation increased albumin (ALB) but reduced creatinine (CRE). Superoxide dismutase, glutathione peroxidase, and catalase activity level increased $(P<0.01)$ with methionine and enzyme supplementation.

Conclusion: Cassava peel meal could be included in growing rabbits' diets at $350 \mathrm{~g} / \mathrm{kg}$ level with methionine at $32.53 \%$ higher than requirement and $0.5-\mathrm{g} / \mathrm{kg}$ multienzyme supplementation.
\end{abstract}

Keywords: Cassava, Wastes, Blood, Anti-oxidative status, Rabbit

\section{Background}

The role played by cassava and its by-products in alleviating food security challenge is tremendous in Africa (Aro, 2008). For the period covering 2008 and 2012, the African continent produced over $54 \%$ of global cassava production, with Nigeria alone contributing 36\% (approximately 52 million tons) of the African total production (FAOStat, 2013). This increased cassava production has offered opportunities to intensify the utilization of cassava by-products such as cassava peels, in animal

\footnotetext{
Correspondence: oloruntoladavid@gmail.com;

olugbenga.oloruntola@aaua.edu.ng

'Animal Science Department, Adekunle Ajasin University, Akungba Akoko, Nigeria

${ }^{2}$ Animal Production Unit, Agricultural Technology Department, The Federal
} Polytechnic, Ado Ekiti, Nigeria

\section{Springer Open}

(c) The Author(s). 2020 Open Access This article is licensed under a Creative Commons Attribution 4.0 International License, which permits use, sharing, adaptation, distribution and reproduction in any medium or format, as long as you give appropriate credit to the original author(s) and the source, provide a link to the Creative Commons licence, and indicate if changes were made. The images or other third party material in this article are included in the article's Creative Commons licence, unless indicated otherwise in a credit line to the material. If material is not included in the article's Creative Commons licence and your intended use is not permitted by statutory regulation or exceeds the permitted use, you will need to obtain permission directly from the copyright holder. To view a copy of this licence, visit http://creativecommons.org/licenses/by/4.0/. weight), when processed, could be used to replace high input-dependent conventional energy feed ingredients in animal production (Oloruntola et al., 2016). However, the high level of structurally indigestible carbohydrates (cellulose, hemicellulose, pectin, and lignin) and high antinutrients (hydrogen cyanide, tannin, and phytate) coupled with low protein content (Oloruntola et al., 2018) have been identified as the major factors limiting its optimal use in monogastric feeding. The negative effects of these limitations on the animals vary from impaired digestibility to poor feed intake culminating in reduced performance (Adegbola and Okonkwo, 2002). For example, cyanide inhibition of the terminal enzyme of the respiratory chain (cytochrome $\mathrm{C}$ oxidase) compromises phosphorylation, and eventual cytotoxic hypoxia is 
expressed in acute cyanide toxicity. In chronic cyanide toxicity, in addition to retarded growth, the increase in serum and urinary levels of thiocyanate is perhaps a continuous cause of sulfur-containing amino acid depletion that takes place in the animal. The thiocyanate also causes inhibition of the intra-thyroidal uptake of iodine, increase in thyroid-stimulating hormone, and a reduction in thyroxine level which is necessary for growth in animals (Lukuyuet al., 2014).

Furthermore, there is a continuous increase in attention given to the preservation of livestock welfare with the aim of enhancing their productivity and preserving them from multi-factorial diseases (Giorgiaet al., 2017). For instance, imbalances in the diet of rabbits have been reported as one of the major causes of deviations from normal physiological functions which usually cause high mortality and low farm productivity (Broom and Johnson, 1993).

Various methods to eliminate or reduce constraints/ limitations to cassava peel utilization in animal feeding have been tried and their accomplished success reported. Hyper methionine supplementation (Oladunjoye et al., 2014, Oloruntola et al., 2019) and exogenous enzyme supplementation (Ogunsipe et al., 2015 Oloruntola 2018, Oloruntola et al., 2019) and fermentation (Oloruntola et al., 2015) have been recommended for enhancing the utilization of cassava peels in monogastric animal feeding. Perhaps sundrying is also another possible effective method of improving the potential feeding value of cassava peel meal.

Sulfur-containing amino acids such as methionine are needed for the detoxifying process of cyanide to thiocyanate. Thus, the determination of amino acids' digestibility in animals fed with cassava by-product-based diet consequently becomes necessary to further monitor the animals' well-being while being fed on these byproducts. Furthermore, there exists an established link between nutrition and oxidative status of animals (Giorgia et al., 2017) and the physiological stress due to imbalances in diets of animals as this could lead to excessive production of free radicals, with resultant oxidative stress (Khadijah et al., 2009). Blood and its constituents can also provide a valuable medium for nutritional and clinical evaluation of the individual because dietary components affect the blood constituents (Onifade \& Tewe, 1993), while serum biochemistry reflects the condition of the organism under the influence of an internal factor such as diet (Akanmu \& Adeyemo, 2012).

Therefore, the present feeding trial was conducted to study the effect of cassava peel meal dietary inclusion, varying methionine levels, and multienzyme supplementation on the digestibility of ileal amino acids, blood composition, and oxidative status of growing rabbits with a view to establishing the safe level of cassava peel meal in the diets of growing rabbits.

\section{Materials and methods}

The approval of protocol for this feeding trial was given by the Research Ethics Committee of Agricultural Technology Department, The Federal Polytechnic, Ado Ekiti, Nigeria. The study was carried out in a well-ventilated pen at the Teaching and Research Farm of Agricultural Technology Department, The Federal Polytechnic, Ado Ekiti, Nigeria. The site was $437 \mathrm{~mm}$ above the sea level and has the mean annual temperature of $26.2^{\circ} \mathrm{C}$ and situated at latitudes of $7^{\circ}$ $37^{\prime} \mathrm{N}$ and $7^{\circ} 12^{\prime} \mathrm{N}$ and longitudes of $5^{\circ} 11^{\prime} \mathrm{E}$ and $5^{\circ} 31^{\prime} \mathrm{E}$ (Oloruntola et al. 2016).

\section{Ingredient preparation and experimental diets}

Cassava peels were collected fresh from a cottage cassava processing factory located at Ado Ekiti, Nigeria, washed with clean water, drained, and spread lightly on a tarpaulin for sun-drying for about 2 weeks from the day of collection. Dried cassava peels were thereafter milled with a 3-mm screen hammer mill to obtain cassava peel meal (CPM) and analyzed for its chemical composition (Table 1). The multienzyme (Biozyme PH) used in this study, according to the manufacturer (Biomix S.A, Carrera 47C, SabanetaColombia), has a minimum per kilogram of cellulase (700,000 U.A), $\alpha$-amylase (800,000 U.A), $\beta$-glucanase (300,000 U.BG), phytase (1200 F.T.U), protease $(8,000$, 000 U.P), lipase (20,000 U.I), and xylanase (500 000 U.X). Eight (8) diets were formulated with their gross composition as shown in Table 2. The eight dietary treatments were pelletized (4-mm diameter and $8-\mathrm{mm}$ long) and designated as diets $1,2,3,4,5,6,7$, and 8 . Diets 1 and 2 had $5.6 \mathrm{~g} / \mathrm{kg}$ (normal methionine level) but only diet 2 was supplemented with exo-enzyme;

Table 1 Composition (\%) of cassava peel meal

\begin{tabular}{ll}
\hline Parameters & Quantity \\
\hline DM & $92.17 \pm 0.49$ \\
Crude protein & $5.48 \pm 0.13$ \\
Crude fiber & $10.50 \pm 0.12$ \\
Ether extract & $0.44 \pm 0.02$ \\
Ash & $5.27 \pm 0.04$ \\
Nitrogen free extract & $70.48 \pm 0.75$ \\
Acid detergent fiber & $13.64 \pm 0.02$ \\
Acid detergent lignin & $2.22 \pm 0.01$ \\
Neutral detergent fiber & $21.94 \pm 0.11$ \\
Methionine & $0.04 \pm 0.00$ \\
Lysine & $0.11 \pm 0.01$ \\
Hydrocyanic acid $(\mathrm{mg} / \mathrm{kg})$ & $20 \pm 0.29$ \\
\hline
\end{tabular}


Table 2 Composition of experimental diets

\begin{tabular}{|c|c|c|c|c|c|c|c|c|}
\hline Ingredients (\%) & Diet 1 & Diet 2 & Diet 3 & Diet 4 & Diet 5 & Diet 6 & Diet 7 & Diet 8 \\
\hline Multienzyme (0.05\%) & - & + & - & + & - & + & - & + \\
\hline DL-methionine 99\% & 0.18 & 0.18 & 0.48 & 0.48 & 0.26 & 0.26 & 0.55 & 0.55 \\
\hline Cassava peel meal & 0.00 & 0.00 & 0.00 & 0.00 & 35.00 & 35.00 & 35.00 & 35.00 \\
\hline Maize & 37.00 & 37.00 & 37.00 & 37.00 & 1.93 & 1.93 & 1.93 & 1.93 \\
\hline BDG & 36.30 & 36.30 & 36.30 & 36.30 & 36.30 & 36.30 & 36.30 & 36.30 \\
\hline Wheat offal & 7.37 & 7.37 & 7.07 & 7.07 & 7.36 & 7.36 & 7.07 & 7.07 \\
\hline Soybean meal & 8.00 & 8.00 & 8.00 & 8.00 & 8.00 & 8.00 & 8.00 & 8.00 \\
\hline Maize husk & 8.00 & 8.00 & 8.00 & 8.00 & 8.00 & 8.00 & 8.00 & 8.00 \\
\hline Bone & 1.00 & 1.00 & 1.00 & 1.00 & 1.00 & 1.00 & 1.00 & 1.00 \\
\hline L-lysine $\mathrm{HCl} 78 \%$ & 0.20 & 0.20 & 0.20 & 0.20 & 0.20 & 0.20 & 0.20 & 0.20 \\
\hline Premix & 0.25 & 0.25 & 0.25 & 0.25 & 0.25 & 0.25 & 0.25 & 0.25 \\
\hline Salt & 0.20 & 0.20 & 0.20 & 0.20 & 0.20 & 0.20 & 0.20 & 0.20 \\
\hline Vegetable oil & 1.00 & 1.00 & 1.00 & 1.00 & 1.00 & 1.00 & 1.00 & 1.00 \\
\hline Titanium dioxide & 0.50 & 0.50 & 0.50 & 0.50 & 0.50 & 0.50 & 0.50 & 0.50 \\
\hline \multicolumn{9}{|c|}{ Determined analysis (\%) } \\
\hline Crude protein & 17.51 & 17.51 & 17.46 & 17.46 & 16.55 & 16.55 & 16.49 & 16.49 \\
\hline Crude fiber & 12.09 & 12.09 & 12.07 & 12.07 & 16.23 & 16.23 & 16.24 & 16.24 \\
\hline Lysine & 0.92 & 0.92 & 0.92 & 0.92 & 0.90 & 0.90 & 0.89 & 0.89 \\
\hline Methionine & 0.58 & 0.58 & 0.85 & 0.85 & 0.59 & 0.59 & 0.85 & 0.85 \\
\hline NDF & 39.96 & 39.96 & 39.94 & 39.94 & 40.12 & 40.12 & 40.14 & 40.14 \\
\hline ADF & 17.54 & 17.54 & 17.36 & 17.36 & 17.92 & 17.92 & 17.95 & 17.95 \\
\hline ADL & 3.61 & 3.61 & 3.62 & 3.62 & 3.94 & 3.94 & 3.96 & 3.96 \\
\hline \multicolumn{9}{|l|}{ Calculated analysis } \\
\hline Crude protein & 17.51 & 17.53 & 17.46 & 17.47 & 16.55 & 16.53 & 16.49 & 16.47 \\
\hline Lysine (\%) & 0.88 & 0.88 & 0.88 & 0.88 & 0.87 & 0.87 & 0.87 & 0.87 \\
\hline Methionine (\%) & 0.56 & 0.56 & 0.83 & 0.83 & 0.55 & 0.55 & 0.81 & 0.81 \\
\hline Calcium (\%) & 0.49 & 0.49 & 0.49 & 0.49 & 0.57 & 0.57 & 0.57 & 0.57 \\
\hline Available P (\%) & 0.35 & 0.35 & 0.36 & 0.36 & 0.39 & 0.39 & 0.39 & 0.39 \\
\hline Energy (kcal/kg) & 2581.5 & 2581.5 & 2577.6 & 2577.6 & 2624.5 & 2624.5 & 2620.7 & 2620.7 \\
\hline *HCN (mg/kg) & 0.0 & 0.0 & 0.0 & 0.0 & 700.0 & 700.0 & 700.0 & 700.0 \\
\hline
\end{tabular}

$B D G$ Brewer's dried grain, NDF neutral detergent fiber, ADF acid detergent fiber, $A D L$ acid detergent lignin, $H C N$ hydrogen cyanide,

*Calculated based on the determined value on Table 1

diets 3 and 4 had $8.3 \mathrm{~g} / \mathrm{kg}$ (higher methionine level), but diet 4 was supplemented with exo-enzyme. In diets 5 and 6, dried cassava peel meal was included at $350 \mathrm{~g} / \mathrm{kg}$ to replace the corresponding amount of maize grain and had $5.6 \mathrm{~g} / \mathrm{kg}$ (normal methionine level), but diet 6 was supplemented with multienzyme. Diets 7 and 8 had their maize grain being replaced by $350 \mathrm{~g} / \mathrm{kg}$ of dried cassava peel meal and had $8.3 \mathrm{~g} / \mathrm{kg}$ (higher methionine level), but diet 8 was multienzyme supplemented.

\section{Animals and experimental design}

A total of 240 crossbred rabbits (Chinchilla $\times$ New Zealand white) of same sex, at 5 weeks of age and average live weight of $572.99 \pm 9.33 \mathrm{~g}$ were randomly allotted to the 8 experimental dietary treatments $(30$ rabbits/dietary treatment; 10 rabbits/replicate; 3 rabbits/replicate). The trial was conducted in a completely randomized design arranged in the form of $2 \times 2 \times 2$ factorial with two CPM levels ( 0 and $350 \mathrm{~g} / \mathrm{kg}), 2$ methionine (MET) levels (5.6 and $8.3 \mathrm{~g} / \mathrm{kg}$ ) and two enzyme (ENZ) levels $(0$ and $0.5 \mathrm{~g} /$ $\mathrm{kg}$ ). Marker (titanium dioxide) was incorporated into each diet at the rate of $0.5 \%$ for the determination of amino acid digestibility.

The rabbits were raised in galvanized wire mesh cages $(60 \times 55 \times 30 \mathrm{~cm}) \mathrm{kept}$ in a well-ventilated pen, provided with fresh water and their respective experimental diets ad libitum throughout the period of the trial (56 days). 


\section{Digestibility study}

On day 56 of the feeding trial, one rabbit from each replicate was randomly selected, tagged, weighed, and sacrificed or rather slaughtered. Immediately, the rabbits were dissected; the ileum contents (digesta) were collected in a clean labeled Petri dish for assessment of amino acid digestibility. The digesta samples were thereafter kept in the deep freezer. To obtain dried samples, the frozen digesta were freeze-dried using $\mathrm{VaCO}_{2}$ Zirbus Technology (Hilfe Gottes, 1-37539, Bad Grund, Germany).

\section{Determination of blood parameters}

At 56 days of age, 10 rabbits from each treatment were randomly selected and bled following procedures described by Burnett et al. (2003). About $8 \mathrm{ml}$ of blood was collected in to tubes from the marginal ear vein of each rabbit with syringe and needle. Each blood sample was divided into two parts, such that $3 \mathrm{ml}$ blood was placed in a potassium ethylene diamine tetraacetic acid (KEDTA) tube for hematological study and the remaining blood placed in the plain (no anticoagulant) tube for serum chemistry and antioxidant enzyme status determination.

\section{Chemical analysis}

The cassava peels and experimental diets were analyzed in triplicates for proximate composition (AOAC, 2000), neutral detergent fiber and acid detergent fiber (Goering and VanSoest, 1970), and cyanide content (Oboh et al., 2002). Amino acid contents of the CPM, diets, and ileal digesta samples were determined as described by Benitez (1989) using Applied Biosystems PTH Amino Acid Analyzer (Applied Biosystems Inc., Foster City, CA, 94404, USA, Model 120A). The $\mathrm{Cr}_{2} \mathrm{O}_{3}$ content of the diets and digesta samples were determined according to the method of Blas et al. (2003), while for the amino acid content, ileal digestibility coefficients were calculated using the following equation:

Digestibility coefficient $=1-\frac{\operatorname{digesta}(\mathrm{g} / \mathrm{kg}) / \operatorname{digesta}_{\mathrm{Cr} 2 \mathrm{O} 3}(\mathrm{~g} / \mathrm{kg})}{\operatorname{diet}(\mathrm{g} / \mathrm{kg}) / \operatorname{digesta}_{\mathrm{Cr} 2 \mathrm{O} 3}(\mathrm{~g} / \mathrm{kg})}$

The hematological indices were determined on the collection day using Shenzhen Mindray Auto Hematology Analyzer (Model Bc-3200, Shenzhen Mindray Biomedical Electronics Co., Hamburg, 20537, Germany). The serum chemistry indices were determined with a Reflectron ${ }^{\circ}$ Plus 8 C79 (Roche Diagnostic, GonbH Mannheim, Germany) using kits. Glutathione peroxidase (GPx) activity in the plasma sample was determined as described by Rotrucket al. (1973), superoxide dismutase (SOD) as described by Misra and
Fridovich (1972), and catalase activity as described by Aebi (1974).

\section{Statistical analysis}

Data generated were subjected to general linear model analysis of variance for factorial $(2 \times 2 \times 2)$ arrangement. First-order and second-order interactions on the main factors were determined. Test for a significant difference between the dietary treatment means was conducted by Duncan's multiple range test at $P \leq 0.05$.

\section{Results}

The effects of cassava peel meal, methionine, and multienzyme supplementation on ileal digestibility of seven (7) amino acids are shown in Table 3. The ileal digestibility of leucine, lysine, and valine was the least $(P<$ $0.05)$ in rabbits fed with diet 5 (350 g/ $/ \mathrm{kg}$ CPM; $5.6 \mathrm{~g} / \mathrm{kg}$ MET) but with the highest methionine digestibility. The CPM inclusion $(350 \mathrm{~g} / \mathrm{kg})$ significantly $(P<0.05)$ reduced the digestibility of leucine ( 0.92 to 0.85 ), lysine ( 0.85 to 0.79 ), valine ( 0.85 to 0.81 ), and threonine ( 0.80 to 0.79 ), while methionine and cysteine digestibility increased ( 0.78 to 0.85 and 0.82 to 0.88 ), respectively. Also in this study, increasing the methionine from $5.6 \mathrm{~g} / \mathrm{kg}$ to $8.3 \mathrm{~g} /$ $\mathrm{kg}$ led to a significant increase in leucine $(P<0.01)$, lysine $(P<0.05)$, and valine $(P<0.01)$ digestibility. Supplemental multienzyme $(0.5 \mathrm{~g} / \mathrm{kg})$ caused significant $(P<$ 0.05 ) percent increase in leucine, lysine, and valine digestibility by $8 \%, 6 \%$, and $3.6 \%$, respectively. Interaction of methionine and enzyme was significant $(P<0.05)$ for leucine and lysine.

Table 4 shows that of all the hematological indices measured, only the lymphocyte was affected. The lymphocyte value recorded for rabbits fed with diet 5 was significantly $(P<0.01)$ lower than the rest of the diets. There was significant $(P<0.03 ; 0.01)$ interactive effects of CPM $\times$ ENZ and MET $\times$ ENZ on lymphocyte count.

There was a significant $(P<0.02)$ reduction of TPR in rabbits fed with diet 5 . In addition, CPM dietary inclusion caused TPR reduction in the rabbits (Table 5), while an increase $(P<0.05)$ in TPR value in the rabbits was recorded due to dietary methionine. There was a significantly $(P<0.01)$ low ALB value recorded in rabbits fed with diet $5(350 \mathrm{~g} / \mathrm{kg} \mathrm{CPM} ; 5.6 \mathrm{~g} / \mathrm{kg} \mathrm{MET})$. However, there was an improvement $(P<0.05)$ in ALB values due to enzyme supplementation.

There was an elevated creatinine level $(P<0.01)$ in rabbits fed with diet $5(350 \mathrm{~g} / \mathrm{kg}$ CPM and $5.6 \mathrm{~g} / \mathrm{kg}$ MET) above other diets, and there was the tendency $(P=0.09)$ of CPM inclusion to numerically increase the creatinine level in this study (Table 5). However, there was a significant $(P<0.05)$ decrease in creatinine levels 
Table 3 Effect of cassava peel meal $(\mathrm{g} / \mathrm{kg})$, methionine $(\mathrm{g} / \mathrm{kg})$, and multienzyme $(\mathrm{g} / \mathrm{kg})$ inclusion on ileal digestibility of some amino acids

\begin{tabular}{|c|c|c|c|c|c|c|c|c|c|c|c|}
\hline Diets & CPM & MET & ENZ & Leu & Lys & Phe & Val & Met & His & Thr & Cyst \\
\hline 1 & 0 & 5.6 & 0.00 & $0.84^{b}$ & $0.78^{b}$ & 0.75 & $0.81^{\mathrm{ab} c}$ & $0.73^{c}$ & 0.78 & 0.77 & 0.76 \\
\hline 2 & 0 & 5.6 & 0.50 & $0.94^{\mathrm{a}}$ & $0.85^{\mathrm{a}}$ & 0.76 & $0.86^{\mathrm{a}}$ & $0.81^{\mathrm{abc}}$ & 0.82 & 0.80 & 0.84 \\
\hline 3 & 0 & 8.3 & 0.00 & $0.93^{\mathrm{a}}$ & $0.85^{\mathrm{a}}$ & 0.78 & $0.86^{a}$ & $0.80^{\mathrm{ab}}$ & 0.82 & 0.82 & 0.84 \\
\hline 4 & 0 & 8.3 & 0.50 & $0.95^{\mathrm{a}}$ & $0.87^{\mathrm{a}}$ & 0.79 & $0.85^{\mathrm{a}}$ & $0.80^{\mathrm{ab}}$ & 0.83 & 0.83 & 0.83 \\
\hline 5 & 350 & 5.6 & 0.00 & $0.69^{d}$ & $0.70^{c}$ & 0.73 & $0.76^{c}$ & $0.91^{\mathrm{a}}$ & 0.75 & 0.75 & 0.93 \\
\hline 6 & 350 & 5.6 & 0.50 & $0.80^{b c}$ & $0.82^{\mathrm{ab}}$ & 0.75 & $0.79^{b c}$ & $0.84^{\mathrm{ab}}$ & 0.80 & 0.78 & 0.86 \\
\hline 7 & 350 & 8.3 & 0.00 & $0.77^{c}$ & $0.81^{\mathrm{ab}}$ & 0.74 & $0.80^{\mathrm{abc}}$ & $0.83^{\mathrm{ab}}$ & 0.79 & 0.78 & 0.85 \\
\hline 8 & 350 & 8.3 & 0.50 & $0.82^{b c}$ & $0.82^{\mathrm{ab}}$ & 0.75 & $0.84^{\mathrm{ab}}$ & $0.83^{\mathrm{ab}}$ & 0.80 & 0.79 & 0.86 \\
\hline SEM & & & & 1.87 & 1.17 & 0.86 & 0.87 & 1.41 & 0.87 & 0.72 & 1.37 \\
\hline$P$ value & & & & 0.01 & 0.01 & 0.74 & 0.01 & 0.05 & 0.58 & 0.11 & 0.14 \\
\hline \multicolumn{12}{|c|}{ Main effect } \\
\hline \multirow[t]{2}{*}{ CPM } & 0 & & & $0.92^{\mathrm{a}}$ & $0.85^{\mathrm{a}}$ & 0.77 & $0.85^{\mathrm{a}}$ & $0.78^{b}$ & 0.82 & $0.80^{\mathrm{a}}$ & $0.82^{\mathrm{b}}$ \\
\hline & 350 & & & $0.78^{b}$ & $0.79^{b}$ & 0.75 & $0.81^{b}$ & $0.85^{\mathrm{a}}$ & 0.79 & $0.78^{b}$ & $0.88^{a}$ \\
\hline SEM & & & & 0.82 & 0.98 & 1.31 & 0.86 & 1.56 & 1.27 & 0.89 & 1.73 \\
\hline$P$ value & & & & 0.01 & 0.01 & 0.18 & 0.01 & 0.01 & 0.16 & 0.03 & 0.03 \\
\hline \multirow[t]{2}{*}{ MET } & & 5.6 & & $0.82^{b}$ & $0.79^{b}$ & 0.75 & $0.81^{b}$ & 0.82 & 0.79 & 0.78 & 0.85 \\
\hline & & 8.3 & & $0.87^{\mathrm{a}}$ & $0.84^{\mathrm{a}}$ & 0.77 & $0.85^{\mathrm{a}}$ & 0.82 & 0.81 & 0.81 & 0.85 \\
\hline SEM & & & & 0.82 & 0.98 & 1.31 & 0.86 & 1.56 & 1.73 & 0.89 & 0.91 \\
\hline$P$ value & & & & 0.01 & 0.03 & 0.32 & 0.01 & 0.93 & 0.31 & 0.07 & 0.88 \\
\hline \multirow[t]{2}{*}{ ENZ } & & & 0.00 & $0.81^{a}$ & $0.79^{\mathrm{a}}$ & 0.75 & $0.81^{\mathrm{a}}$ & 0.81 & 0.79 & 0.78 & 0.85 \\
\hline & & & 0.50 & $0.88^{b}$ & $0.84^{b}$ & 0.77 & $0.84^{\mathrm{b}}$ & 0.82 & 0.81 & 0.80 & 0.86 \\
\hline SEM & & & & 0.82 & 0.98 & 1.31 & 0.86 & 1.56 & 1.27 & 0.89 & 1.73 \\
\hline$P$ value & & & & 0.01 & 0.02 & 0.45 & 0.05 & 0.88 & 0.22 & 0.11 & 0.89 \\
\hline \multicolumn{12}{|c|}{ Interactions } \\
\hline \multicolumn{12}{|c|}{$\mathrm{CPM} \times \mathrm{MET}$} \\
\hline SEM & & & & 1.17 & 1.39 & 1.85 & 1.22 & 2.21 & 1.79 & 1.26 & 2.44 \\
\hline$P$ value & & & & 0.92 & 0.71 & 0.51 & 0.31 & 1.11 & 0.99 & 0.35 & 1.38 \\
\hline \multicolumn{12}{|c|}{$C P M \times E N Z$} \\
\hline SEM & & & & 1.17 & 1.39 & 1.85 & 1.22 & 2.21 & 1.79 & 1.26 & 2.44 \\
\hline$P$ value & & & & 0.32 & 0.54 & 0.79 & 0.55 & 0.09 & 0.91 & 0.84 & 0.16 \\
\hline \multicolumn{12}{|c|}{$\mathrm{MET} \times \mathrm{ENZ}$} \\
\hline SEM & & & & 1.17 & 1.39 & 1.85 & 1.22 & 2.21 & 1.79 & 1.26 & 2.44 \\
\hline$P$ value & & & & 0.01 & 0.01 & 0.73 & 0.46 & 0.88 & 0.39 & 0.37 & 0.89 \\
\hline \multicolumn{12}{|c|}{$\mathrm{CPM} \times \mathrm{MET} \times \mathrm{ENZ}$} \\
\hline SEM & & & & 1.64 & 1.97 & 2.61 & 1.72 & 3.13 & 2.54 & 1.78 & 3.46 \\
\hline$P$ value & & & & 0.81 & 0.41 & 0.92 & 0.22 & 0.10 & 0.90 & 0.95 & 0.13 \\
\hline
\end{tabular}

CPM cassava peel meal, MET methionine, ENZ enzyme, Leu leucine, Lys lysine, Iso isoleucine, Phe phenylalanine, Val valine, Met methionine, His histidine, Thr threonine, Cyst cystein, a b, c Means with a different superscript in the same column are significantly different $(P<0.05)$

of the rabbits by high methionine and multienzyme supplementation.

Bilirubin level was significantly $(P<0.05)$ higher in rabbits fed with diet $5(350 \mathrm{~g} / \mathrm{kg}$ CPM and $5.6 \mathrm{~g} / \mathrm{kg}$ MET) compared to those fed the with other diets. In addition, the bilirubin level increased significantly $(P<$
$0.01)$ with $\mathrm{CPM}(350 \mathrm{~g} / \mathrm{kg})$ inclusion but reduced $(P<$ 0.01 ) with methionine supplementation (Table 5). Reduced $(P<0.05)$ bilirubin level caused by high methionine $(8.3 \mathrm{~g} / \mathrm{kg})$ supplementation and significant $(P<0.01)$ interactive effect of CPM and MET was also recorded in this study. 
Table 4 Effect of cassava peel meal $(\mathrm{g} / \mathrm{kg})$, methionine $(\mathrm{g} / \mathrm{kg})$, and multi-enzyme $(\mathrm{g} / \mathrm{kg})$ inclusion on hematological and erythrocytic indices in rabbits

\begin{tabular}{|c|c|c|c|c|c|c|c|c|c|c|c|c|}
\hline Diets & CPM & MET & ENZ & PCV & $\mathrm{HB}$ & RBC & MCV & $\mathrm{MCH}$ & $\mathrm{MCHC}$ & WBC & LYM & PLA \\
\hline 1 & 0 & 5.6 & 0.00 & 35.77 & 12.68 & 7.46 & 50.64 & 17.70 & 35.42 & 7.33 & $1.59^{\mathrm{bc}}$ & 328.33 \\
\hline 2 & 0 & 5.6 & 0.50 & 37.09 & 13.23 & 8.66 & 46.20 & 16.20 & 35.55 & 7.49 & $1.74^{\mathrm{abc}}$ & 323.66 \\
\hline 3 & 0 & 8.3 & 0.00 & 37.14 & 12.75 & 8.93 & 55.16 & 18.10 & 34.98 & 7.78 & $2.38^{\mathrm{a}}$ & 320.32 \\
\hline 4 & 0 & 8.3 & 0.50 & 37.73 & 12.82 & 9.02 & 52.33 & 16.58 & 34.41 & 7.88 & $1.72^{\mathrm{abc}}$ & 317.67 \\
\hline 5 & 350 & 5.6 & 0.00 & 34.17 & 12.56 & 4.07 & 86.54 & 32.18 & 36.54 & 7.08 & $1.09^{c}$ & 299.00 \\
\hline 6 & 350 & 5.6 & 0.50 & 34.37 & 12.90 & 5.27 & 66.42 & 24.32 & 37.39 & 7.26 & $2.01^{\mathrm{ab}}$ & 324.33 \\
\hline 7 & 350 & 8.3 & 0.00 & 34.49 & 12.91 & 5.34 & 64.94 & 23.77 & 37.409 & 7.28 & $2.02^{\mathrm{ab}}$ & 322.66 \\
\hline 8 & 350 & 8.3 & 0.50 & 34.57 & 12.94 & 6.10 & 61.85 & 22.48 & 37.02 & 7.33 & $2.01^{\mathrm{ab}}$ & 314.00 \\
\hline SEM & & & & 0.84 & 0.44 & 0.61 & 4.68 & 1.59 & 0.99 & 0.22 & 0.11 & 318.75 \\
\hline$P$ value & & & & 0.94 & 1.00 & 0.29 & 0.53 & 0.15 & 0.99 & 0.99 & 0.01 & 0.99 \\
\hline \multicolumn{13}{|c|}{ Main effect } \\
\hline \multirow[t]{2}{*}{ CPM } & 0.00 & & & 36.93 & 12.87 & $8.52^{\mathrm{a}}$ & $17.15^{\mathrm{b}}$ & 51.08 & 35.10 & 7.62 & 1.86 & 322.50 \\
\hline & 350 & & & 34.40 & 12.83 & $5.19^{\mathrm{b}}$ & $25.69^{a}$ & 69.94 & 37.09 & 6.74 & 1.78 & 325.00 \\
\hline SEM & & & & 1.35 & 0.75 & 0.83 & 2.02 & 6.74 & 1.64 & 0.37 & 0.11 & 13.51 \\
\hline$P$ value & & & & 0.21 & 0.96 & 0.01 & 0.01 & 0.06 & 0.40 & 0.47 & 0.59 & 0.70 \\
\hline \multirow[t]{2}{*}{ MET } & & 5.6 & & 35.35 & 12.84 & 6.36 & 22.60 & 62.45 & 36.22 & 6.79 & $1.61^{\mathrm{b}}$ & 318.83 \\
\hline & & 8.3 & & 35.98 & 12.85 & 7.34 & 20.23 & 58.57 & 35.95 & 7.57 & $2.03^{\mathrm{a}}$ & 318.66 \\
\hline SEM & & & & 1.35 & 0.75 & 0.83 & 2.02 & 6.74 & 1.64 & 0.37 & 0.11 & 13.51 \\
\hline$P$ value & & & & 0.74 & 0.99 & 0.41 & 0.42 & 0.69 & 0.91 & 0.15 & 0.01 & 0.99 \\
\hline \multirow[t]{2}{*}{ ENZ } & & & 0.00 & 35.39 & 12.72 & 6.45 & 22.94 & 64.32 & 36.08 & 6.87 & 1.77 & 317.58 \\
\hline & & & 0.50 & 35.94 & 12.97 & 7.26 & 19.90 & 56.70 & 36.10 & 7.49 & 1.87 & 319.91 \\
\hline SEM & & & & 1.35 & 0.75 & 0.83 & 2.02 & 6.74 & 1.64 & 0.37 & 0.11 & 13.51 \\
\hline$P$ value & & & & 0.78 & 0.82 & 0.49 & 0.30 & 0.43 & 0.99 & 0.24 & 0.49 & 0.90 \\
\hline \multicolumn{13}{|c|}{ Interactions } \\
\hline \multicolumn{13}{|c|}{$\mathrm{CPM} \times \mathrm{MET}$} \\
\hline SEM & & & & 1.91 & 1.06 & 1.17 & 2.86 & 9.54 & 2.33 & 0.52 & 0.15 & 19.11 \\
\hline$P$ value & & & & 0.84 & 0.86 & 0.95 & 0.34 & 0.34 & 0.82 & 0.49 & 0.79 & 0.72 \\
\hline \multicolumn{13}{|c|}{$C P M \times E N Z$} \\
\hline SEM & & & & 1.91 & 1.06 & 1.17 & 2.86 & 9.54 & 2.33 & 0.52 & 0.15 & 19.11 \\
\hline$P$ value & & & & 0.83 & 0.95 & 0.88 & 0.59 & 0.68 & 0.92 & 0.36 & 0.02 & 0.75 \\
\hline \multicolumn{13}{|c|}{$\mathrm{MET} \times \mathrm{ENZ}$} \\
\hline SEM & & & & 1.91 & 1.06 & 1.17 & 2.86 & 9.54 & 2.33 & 0.52 & 0.15 & 19.11 \\
\hline$P$ value & & & & 0.91 & 0.85 & 0.74 & 0.57 & 0.63 & 0.83 & 0.31 & 0.01 & 0.68 \\
\hline \multicolumn{13}{|c|}{$\mathrm{CPM} \times \mathrm{MET} \times \mathrm{ENZ}$} \\
\hline SEM & & & & 2.71 & 1.50 & 1.65 & 4.04 & 13.49 & 3.29 & 0.74 & 0.21 & 27.02 \\
\hline$P$ value & & & & 0.93 & 0.96 & 0.88 & 0.57 & 0.69 & 0.95 & 0.33 & 0.83 & 0.64 \\
\hline
\end{tabular}

CPM cassava peel meal, MET methionine, ENZ enzyme, $P C V$ packed cell volume (\%), HB hemoglobin concentration ( $\mathrm{g} / \mathrm{dl}), R B C$ red blood cells $\left(\times 10^{12} /\right)$ ), $M C H$ mean cell hemoglobin $(\mathrm{pg}), M C V$ mean cell volume $(\mathrm{fl}), M C H C$ mean cell hemoglobin concentration $(\mathrm{g} / \mathrm{dl})$, WBC white blood cells $\left(\times 10^{9} / \mathrm{l}\right), L Y M$ lymphocytes $\left(\times 10^{9} / \mathrm{l}\right), P L A$ platelets $\left(\times 10^{9} /\right)$, a , c c Means with a different superscript in the same column are significantly different $(P<0.05)$

Alanine aminotransferase (ALT) was stable $(P>0.05)$ across the diets with the highest and the lowest values recorded in rabbits fed with diets 5 and 1, respectively. The CPM inclusion significantly $(P<0.05)$ increased ALT levels in the rabbits. Aspartate aminotransferase (AST) level was higher $(P<0.05)$ in rabbits fed with diet
5 than others (Table 5). In addition, AST level increased $(P<0.05)$ with CPM inclusion in the diet but numerically decreased with high methionine supplementation. $\mathrm{CPM} \times$ MET interaction was significant for AST.

The levels of antioxidant defense enzymes (superoxide dismutase, glutathione peroxidase, and catalase) studied 
Table 5 Effect of cassava peel meal $(\mathrm{g} / \mathrm{kg})$, methionine $(\mathrm{g} / \mathrm{kg})$, and multienzyme $(\mathrm{g} / \mathrm{kg})$ inclusion on serum biochemical indices in rabbits

\begin{tabular}{|c|c|c|c|c|c|c|c|c|c|c|c|c|}
\hline Diets & CPM & MET & ENZ & TPR & ALB & GLO & CRE & URE & $\mathrm{CHO}$ & BIL & $\mathrm{ALT}$ & AST \\
\hline 1 & 0 & 5.6 & 0.00 & $6.61^{a}$ & $2.80^{\mathrm{a}}$ & 3.27 & $73.00^{b}$ & 5.64 & 1.19 & $5.55^{d}$ & 45.01 & $49.87^{b}$ \\
\hline 2 & 0 & 5.6 & 0.50 & $6.21^{\mathrm{a}}$ & $2.85^{\mathrm{a}}$ & 3.32 & $70.10^{b}$ & 5.57 & 1.07 & $5.20^{d}$ & 49.80 & $46.50^{b}$ \\
\hline 3 & 0 & 8.3 & 0.00 & $6.35^{\mathrm{a}}$ & $2.97^{\mathrm{a}}$ & 3.38 & $73.35^{b}$ & 5.99 & 1.08 & $5.42^{d}$ & 48.87 & $47.01^{b}$ \\
\hline 4 & 0 & 8.3 & 0.50 & $6.40^{\mathrm{a}}$ & $2.97^{\mathrm{a}}$ & 3.43 & $72.50^{b}$ & 6.01 & 1.13 & $5.43^{d}$ & 48.62 & $54.41^{\mathrm{b}}$ \\
\hline 5 & 350 & 5.6 & 0.00 & $4.37^{\mathrm{b}}$ & $2.14^{\mathrm{b}}$ & 2.32 & $132.71^{\mathrm{a}}$ & 4.99 & 1.03 & $13.26^{\mathrm{a}}$ & 73.86 & $96.92^{\mathrm{a}}$ \\
\hline 6 & 350 & 5.6 & 0.50 & $6.14^{\mathrm{a}}$ & $2.75^{\mathrm{a}}$ & 3.39 & $73.85^{b}$ & 5.47 & 1.21 & $11.33^{\mathrm{ab}}$ & 66.05 & $57.82^{\mathrm{b}}$ \\
\hline 7 & 350 & 8.3 & 0.00 & $6.15^{\mathrm{a}}$ & $2.81^{a}$ & 3.34 & $70.58^{b}$ & 5.82 & 1.13 & $8.72^{b c}$ & 61.35 & $55.85^{\mathrm{b}}$ \\
\hline 8 & 350 & 8.3 & 0.50 & $6.29^{a}$ & $2.86^{\mathrm{a}}$ & 3.30 & $68.13^{b}$ & 6.00 & 1.15 & $7.66^{\mathrm{cd}}$ & 58.09 & $52.05^{b}$ \\
\hline SEM & & & & 0.16 & 0.06 & 0.11 & 5.34 & 0.14 & 0.07 & 0.65 & 3.75 & 4.05 \\
\hline$P$ value & & & & 0.02 & 0.01 & 0.31 & 0.01 & 0.71 & 0.99 & 0.01 & 0.54 & 0.01 \\
\hline \multicolumn{13}{|c|}{ Main effect } \\
\hline \multirow[t]{2}{*}{ CPM } & 0 & & & $6.28^{\mathrm{a}}$ & $2.89^{a}$ & 3.35 & 72.23 & 5.80 & 1.12 & $5.40^{\mathrm{b}}$ & $48.07^{b}$ & $49.44^{b}$ \\
\hline & 350 & & & $5.74^{\mathrm{b}}$ & $2.64^{b}$ & 3.08 & 86.08 & 5.57 & 1.13 & $10.24^{a}$ & $64.83^{\mathrm{a}}$ & $65.66^{\mathrm{a}}$ \\
\hline SEM & & & & 0.18 & 0.05 & 0.16 & 5.54 & 0.21 & 0.11 & 0.44 & 5.41 & 4.21 \\
\hline$P$ value & & & & 0.05 & 0.01 & 0.26 & 0.09 & 0.46 & 0.94 & 0.01 & 0.04 & 0.02 \\
\hline \multirow[t]{2}{*}{ MET } & & 5.6 & & $5.72^{b}$ & $2.63^{b}$ & 3.07 & $86.43^{a}$ & 5.41 & 1.13 & $8.83^{\mathrm{a}}$ & 58.68 & 62.78 \\
\hline & & 8.3 & & $6.30^{\mathrm{a}}$ & $2.90^{\mathrm{a}}$ & 3.36 & $70.24^{b}$ & 5.95 & 1.12 & $6.80^{b}$ & 54.23 & 52.33 \\
\hline SEM & & & & 0.18 & 0.05 & 0.16 & 5.54 & 5.34 & 0.11 & 0.44 & 5.41 & 4.21 \\
\hline$P$ value & & & & 0.03 & 0.01 & 0.22 & 0.05 & 0.10 & 0.95 & 0.01 & 0.56 & 0.09 \\
\hline \multirow[t]{2}{*}{ ENZ } & & & 0.00 & 5.76 & $2.68^{b}$ & 3.08 & $87.41^{a}$ & 5.61 & 1.11 & 8.23 & 57.27 & 62.41 \\
\hline & & & 0.50 & 6.26 & $2.85^{\mathrm{a}}$ & 3.36 & $71.14^{b}$ & 5.76 & 1.14 & 7.40 & 55.64 & 52.69 \\
\hline SEM & & & & 0.18 & 0.05 & 0.16 & 5.54 & 0.21 & 0.11 & 0.44 & 5.41 & 4.21 \\
\hline$P$ value & & & & 0.06 & 0.05 & 0.23 & 0.05 & 0.62 & 0.84 & 0.21 & 0.83 & 0.12 \\
\hline \multicolumn{13}{|c|}{ Interactions } \\
\hline \multicolumn{13}{|c|}{$\mathrm{CPM} \times \mathrm{MET}$} \\
\hline SEM & & & & 0.25 & 0.08 & 0.23 & 7.83 & 0.31 & 0.16 & 0.63 & 7.65 & 5.96 \\
\hline$P$ value & & & & 0.15 & 0.15 & 0.45 & 0.04 & 0.65 & 0.89 & 0.01 & 0.46 & 0.05 \\
\hline \multicolumn{13}{|c|}{$\mathrm{CPM} \times \mathrm{ENZ}$} \\
\hline SEM & & & & 0.25 & 0.08 & 0.23 & 7.83 & 0.31 & 0.16 & 0.63 & 7.65 & 5.96 \\
\hline$P$ value & & & & 0.09 & 0.08 & 0.32 & 0.09 & 0.57 & 0.69 & 0.31 & 0.61 & 0.06 \\
\hline \multicolumn{13}{|c|}{ MET $\times$ ENZ } \\
\hline SEM & & & & 0.25 & 0.08 & 0.23 & 7.83 & 0.31 & 0.16 & 0.63 & 7.65 & 5.96 \\
\hline$P$ value & & & & 0.13 & 0.08 & 0.24 & 0.08 & 0.86 & 0.98 & 0.63 & 0.98 & 0.07 \\
\hline \multicolumn{13}{|c|}{$\mathrm{CPM} \times \mathrm{MET} \times \mathrm{ENZ}$} \\
\hline SEM & & & & 0.36 & 0.12 & 0.32 & 11.07 & 0.43 & 0.23 & 0.89 & 10.83 & 8.43 \\
\hline$P$ value & & & & 0.13 & 0.13 & 0.24 & 0.10 & 0.75 & 0.63 & 0.84 & 0.75 & 0.31 \\
\hline
\end{tabular}

CPM cassava peel meal, MET methionine, ENZ enzyme, TPR total protein $(\mathrm{g} / \mathrm{dl}), A L B$ albumin $(\mathrm{g} / \mathrm{dl})$, GLO globulin ( $\mathrm{g} / \mathrm{dl}), C R E \mathrm{creatinine}(\mu \mathrm{mol} / \mathrm{l}), U R E \mathrm{urea}(\mathrm{mmol} / \mathrm{l})$, CHO cholesterol $(\mathrm{mmol} / \mathrm{l}), B / L$ bilirubin $(\mu \mathrm{mol} / \mathrm{l}), A L T$ alanine amino transferase $(\mathrm{u} / \mathrm{l})$, AST aspertate amino transferase $(\mathrm{u} / \mathrm{l}), \mathrm{ab}, \mathrm{cd}$, Means with a different superscript in the same column are significantly different $(P<0.05)$

were least $(P<0.01)$ in rabbits fed with diet 5 (Table 6). In addition, these enzyme levels decreased significantly $(P<0.05)$ with $C P M$ inclusion but increased with high methionine and multienzyme supplementation. Generally, there were significant interactive effects of the CPM, MET, and ENZ.

\section{Discussion}

The result of this study showed that the ileal digestibility of leucine, lysine, and valine was least in rabbits fed with diet 5 (350 g/kg CPM; $5.6 \mathrm{~g} / \mathrm{kg}$ MET) but with the highest methionine digestibility. The cyanogenic activity of CPM-based diets (Table 2) may be responsible for the 
Table 6 Effect of cassava peel $(\mathrm{g} / \mathrm{kg})$, methionine $(\mathrm{g} / \mathrm{kg})$, and multienzyme $(\mathrm{g} / \mathrm{kg})$ inclusion on antioxidant status in rabbits

\begin{tabular}{lllllll}
\hline Diets & CPM & MET & ENZ & SOD $(\%)$ & GPx $(\mu \mathrm{g} / \mathrm{g})$ & Catalase $(\mathrm{mM} / \mathrm{ml} / \mathrm{min})$ \\
\hline 1 & 0 & 5.6 & 0.00 & $82.33^{\mathrm{b}}$ & $126.58^{\mathrm{b}}$ & $11.38^{\mathrm{b}}$ \\
2 & 0 & 5.6 & 0.50 & $88.33^{\mathrm{ab}}$ & $165.62^{\mathrm{a}}$ & $13.70^{\mathrm{a}}$ \\
3 & 0 & 8.3 & 0.00 & $86.67^{\mathrm{a}}$ & $166.68^{\mathrm{a}}$ & $14.59^{\mathrm{a}}$ \\
4 & 0 & 8.3 & 0.50 & $92.33^{\mathrm{a}}$ & $168.81^{\mathrm{a}}$ & $14.73^{\mathrm{a}}$ \\
5 & 350 & 5.6 & 0.00 & $64.67^{\mathrm{c}}$ & $100.15^{\mathrm{c}}$ & $8.77^{\mathrm{c}}$ \\
6 & 350 & 5.6 & 0.50 & $87.00^{\mathrm{ab}}$ & $162.74^{\mathrm{a}}$ & $13.67^{\mathrm{a}}$ \\
7 & 350 & 8.3 & 0.00 & $87.32^{\mathrm{ab}}$ & $163.20^{\mathrm{a}}$ & $14.53^{\mathrm{a}}$ \\
8 & 350 & 8.3 & 0.50 & $90.65^{\mathrm{a}}$ & $162.83^{\mathrm{a}}$ & $14.71^{\mathrm{a}}$ \\
SEM & & & & 1.80 & 4.91 & 0.42 \\
$P$ value & & & 0.01 & 0.01 & 0.01
\end{tabular}

Main effect

\begin{tabular}{|c|c|c|c|c|c|}
\hline \multirow[t]{2}{*}{ CPM } & & & $87.91^{\mathrm{a}}$ & $156.92^{\mathrm{a}}$ & $13.60^{\mathrm{a}}$ \\
\hline & & & $82.41^{b}$ & $147.23^{b}$ & $12.92^{b}$ \\
\hline SEM & & & 0.94 & 1.03 & 0.17 \\
\hline$P$ value & & & 0.01 & 0.01 & 0.01 \\
\hline \multirow[t]{2}{*}{ MET } & 5.6 & & $80.58^{b}$ & $138.77^{b}$ & $11.88^{\mathrm{b}}$ \\
\hline & 8.3 & & $89.75^{a}$ & $165.38^{\mathrm{a}}$ & $14.64^{\mathrm{a}}$ \\
\hline SEM & & & 0.94 & 1.03 & 0.17 \\
\hline$P$ value & & & 0.01 & 0.01 & 0.01 \\
\hline \multirow[t]{2}{*}{ ENZ } & & 0.00 & $80.75^{b}$ & $139.15^{b}$ & $12.32^{b}$ \\
\hline & & 0.50 & $89.58^{a}$ & $165.00^{\mathrm{a}}$ & $14.20^{\mathrm{a}}$ \\
\hline SEM & & & 0.94 & 1.03 & 0.17 \\
\hline$P$ value & & & 0.01 & 0.01 & 0.01 \\
\hline
\end{tabular}

Interactions

CPM*MET

$\begin{array}{lccc}\text { SEM } & 1.34 & 1.45 & 0.24 \\ P \text { value } & 0.01 & 0.00 & 0.02 \\ \text { CPM*ENZ } & & & \\ \text { SEM } & 1.34 & 1.45 & 0.24 \\ P \text { value } & 0.01 & 0.00 & 0.02 \\ \text { MET*ENZ } & & & \\ \text { SEM } & 1.34 & 1.45 & 0.24 \\ P \text { value } & 0.01 & 0.00 & 0.00 \\ \text { CPM*MET*ENZ } & & & \\ \text { SEM } & 1.89 & 2.06 & 0.33 \\ P \text { value } & 0.01 & 0.00 & 0.02\end{array}$

CPM cassava peel meal $(\mathrm{g} / \mathrm{kg}), M E T$ methionine $(\mathrm{g} / \mathrm{kg}), E N Z$ enzyme $(\mathrm{g} / \mathrm{kg}), S O D$ superoxide dismutase, GPx glutathione peroxidase, ${ }^{a}, \mathrm{~b}, \mathrm{c}$ Means with a different superscript in the same column are significantly different $(P<0.05)$

decrease in the digestibility of these amino acids as recorded in this study. This findings agreed with the previous report of Iyayi and Odueso (2003) who recorded low nutrient digestibility following varying cyanide levels in rabbit diets. However, the observed high methionine and cysteine (sulfur-containing amino acids) digestibility recorded in this study following CPM inclusion may be attributed to the fact that sulfur from these amino acids could have been highly used in the process of detoxification of cyanide in the CPM-based diets by the experimental rabbits. According to Morgan and Choct (2016), sulfur-consuming processes involved in the detoxification process of hydrocyanic acid varied from the liberation of hydrocyanic acid by glucosidases being produced by the intestinal microbiota, liver, and other tissues. In the liver, hydrocyanic acid is changed into thiocyanate by rhodanese and thereafter excreted in the urine (Garcia \& Dale, 1999). In addition, the use of these sulfur amino acids for the purpose of cyanide detoxification makes them unavailable for protein synthesis in animals. This may aggravate the deficiency of these amino acids, coupled with the thermal stress involved in amino acid catabolism; this could lead to the reduction in digestibility of other amino acids (Iyayi \& Odueso, 2003) as experienced in this study. Also in this study, the observed increased digestibility of leucine, lysine, and valine due to increased methionine level from $5.6 \mathrm{~g} / \mathrm{kg}$ to $8.3 \mathrm{~g} / \mathrm{kg}$ is in tandem with the report of Oladunjoye et al. (2014) who observed improvement in nutrient utilization in broilers fed with cassava-based diet supplemented with high methionine level. Improvement in amino acid digestibility due to methionine level may also be associated with the detoxifying effect of methionine on hydrocyanic acid (Aniebo, 2012) which could probably reduce the negative impacts of cyanide on nutrient digestibility efficiency. The increase of leucine, lysine, and valine digestibility due to supplemental multienzyme recorded in this study is consistent with the recent reports of Hossain et al. (2016) and Ayodele et al. (2016) who both recorded an improvement in the digestibility of nutrients due to enzyme supplementation. The enzyme enables the animals to degrade antinutrients in feed components (Kiarieet al., 2013; Oloruntola et al., 2019), promotes breakdown of stored proteins by increasing the degree of protein hydrolysis, increasing the proportion of low molecular size proteins, and promoting the availability of protein for uptake (Frietas et al., 2011; Ayodele et al., 2016). This may in part explain the reason behind the improvement in amino acid digestibility due to multienzyme supplementation in this study. The significant interaction effect MET $\times$ ENZ on leucine and lysine implies that these two factors would be able to complement one another to bring about improvement in the digestibility of these amino acids in a growing rabbit.

Lymphocytes play a vital role in the body defense of animals against infections. In particular, $T$ cells, which are a group of lymphocyte cells responsible for cellmediated immunity had been reported to be affected by nutrition and any factor that cause gastrointestinal 
malabsorption (Dennis, 2005). Roles of nutrition in preserving or increasing the T-lymphocytes or CD4 cells were reported (Okorie \& Ophori, 2013). The decrease in the lymphocyte value in rabbits fed with diet $5(350 \mathrm{~g} / \mathrm{kg}$ CPM) may be the resultant effect of a relatively high level of $\mathrm{HCN}$ in the diet coupled with low methionine and no multienzyme addition which possibly could lead to the impairment/prevention of essential nutrient digestion and utilization (Prathibha et al, 1995, Lukuyu et al., 2014). This is explained further by the increase of lymphocyte count due to high-level methionine supplementation in this study. Methionine supplementation was reported to improve nutrient utilization and growth performance in rabbits (Job, 1975; Oloruntola et al., 2019). The significant interactive effects of CPM $\times$ ENZ and MET $\times$ ENZ on lymphocyte count revealed the combining positive effect of these factors on the immunity of the experimental rabbits. However, all the hematological and derived absolute values recorded in this study fall within the normal range (PCV 33-50\%, Hb 9.4-17.4 g/dl, RBC 4.7-7.2 $\times 10^{12} / \mathrm{l}$, MCV 50-75 fl, MCH 16-23 pg and MCHC 280-360 g/l, WBC $5-12 \times 10^{9} / 1$, lymphocytes $2-20 \times 10^{9} / 1$, platelets $200-1000 \times 10^{9} / \mathrm{l}$ ) as earlier reported (Burnet et al., 2003; Latimer et al., 2003 and Oloruntola et al., 2016).

Total protein (TPR), albumin (ALB), and globulin (GLO) are indicators for measuring dietary protein intake and utilization by animals (Onifade \& Tewe, 1993). In this study, the reduction of TPR in rabbits fed with diet 5 and the observed TPR reduction in the rabbits due to CPM dietary inclusions indicate the possible impairment of protein intake in rabbits due to the presence of $\mathrm{HCN}$, the major antinutrient present in CPM. However, the increase in TPR value in the rabbits due to dietary methionine further unveiled the tendency of methionine supplementation to improve the nutritive values of CPM-based diets in rabbit nutrition. In the same vein, low ALB value recorded in rabbits fed with diet $5(350 \mathrm{~g} / \mathrm{kg}$ CPM; 5.6 $\mathrm{g} / \mathrm{kg}$ MET) could be associated with nutritional imbalances precipitated relatively by high dietary $\mathrm{HCN}$ concentration caused by the inclusion of CPM in the diet. This could be further explained by the reduction of ALB value in the experimental rabbits due to CPM dietary inclusion. However, the observed improvement in ALB values due to enzyme supplementation further showed the efficiency of the multienzyme supplementation in improving the nutrient utilization and reduction of negative effects of antinutrients in farm animals. In general, the present findings further confirm the toxicity effect of cyanide, which could lead to decrease protein production and increased loss or catabolism of protein with resultant decreased concentration of TPR and decreased production of fibrinogen, immunoglobin, and globulins or increased loss of albumin which also might have resulted in decreased ALB concentration (Lording \& Friend, 1991).

In addition, the improvement of TPR and ALB concentrations in rabbits fed with diets containing high methionine and multienzyme supplementation agreed with the reports of Oladunjoye et al. (2014), Ogunsipe et al. (2015), and Oloruntola (2018) who separately reported improvement in the nutritional value of cassava peel meal supplemented with methionine and exo-enzyme. Increased rhodanese activity in kidney and excretion of cyanide metabolites in urine (Wrobel et al., 2004) are among the responses to cyanide poisoning in animals' body systems, meaning that kidneys play some roles in cyanide detoxification. Therefore, the elevation of creatinine levels in rabbits fed with diet $5(350 \mathrm{~g} / \mathrm{kg}$ CPM and $5.6 \mathrm{~g} / \mathrm{kg} \mathrm{MET)}$ above other diets and the tendency of CPM inclusion to increase the creatinine level in this study signals a compromise in the integrity of the rabbits' kidney cell due to the presence of cyanide. However, the decrease in creatinine levels of the rabbits by high methionine and multienzyme supplementation in this study further supports the earlier reports on the potentials of methionine (Oladunjoye et al., 2014) and multienzyme (Ogunsipe et al., 2015) in removing the negative effect of cyanide present in cassava by-productbased diet in animals.

Bilirubin level reflects the balance between its production and excretion. Since cyanide detoxification is a function of the liver (Nakajima, 2015), the elevated bilirubin level in rabbits fed with diet 5 in this study may reflect possible liver disorder precipitated by cyanide in the diet due to CPM inclusion. However, the observed reduced bilirubin level caused by high methionine $(8.3 \mathrm{~g} /$ $\mathrm{kg}$ ) supplementation in this study could be linked to the activities of rhodanese (the key enzyme involved in sulfur metabolism) which use methionine as a sulfur donor to detoxify cyanide to thiocyanate (Nakajima, 2015). This is further supported by the significant interactive effect of CPM and MET in this study; implying that CPM and MET could interact to produce a positive effect on the health status of the experimental rabbits.

The increased ALT levels in the rabbits due to CPM dietary inclusion also indicate possible hepatocellular injury in the rabbits caused by hydrogen cyanide. However, high methionine and multienzyme supplementation brought the numeric decrease in ALT levels. AST is an important heart status marker. Generally, the result from this study showed that CPM inclusion in rabbits' diets may compromise the liver and heart integrity, but methionine and multienzyme supplementation as shown in this current study may avert this possible health mishap. All the serum chemistry values shown in Table 5 fall within the normal range (total protein $2.8-10.0 \mathrm{~g} / \mathrm{dl}$, albumin $3.3-5 \mathrm{~g} / \mathrm{dl}$, globulin $1.5-2.7 \mathrm{~g} / \mathrm{dl}$, creatinine $70-150 \mu \mathrm{mol} /$ 
l, bilirubin 4.3-12.8 $\mu \mathrm{mol} / \mathrm{l}$, urea $9.3-25.3 \mathrm{mmol} / \mathrm{l}$, cholesterol 0.1-2.0 mmol/l, ALT 55-260 u/l, and AST 33-99 u/ l) reported earlier for rabbits (Burnet et al., 2003).

Cyanide caused lipid oxidation in varieties of tissues and imposes oxidative stress on rabbits by inhibition of antioxidant defense enzyme that catalyzes the metabolism of oxygen radicals and peroxides (Shouet al., 2000; Gary \& Gary, 2015). It also inhibits metalloenzymes which makes up the anti-oxidation defense (superoxide dismutase, catalase, glutathione peroxidase, and glutathione reductase) (Gary \& Gari, 2015). This might suggest the observed decrease in the values of the superoxide dismutase, catalase, glutathione peroxidase, and glutathione reductase in rabbits fed with diet 5 . The enzyme (carbohydrase) can increase the permeability of the aleurone layer and consequently increase the release of unavailable minerals to the animals. For instance, selenium is involved in the active site of enzyme glutathione peroxidase in blood, liver, and edible tissues (Surai, 2002). Therefore, the improvement in the anti-oxidative status of the rabbits due to multienzyme supplementation could be a result of the direct effect of these enzymes or the combined interactive effect of the component enzyme in the multienzyme used in releasing useful minerals that promote the production of antioxidantprotective enzymes in the experimental rabbits. Methionine is a precursor of cysteine, which is required for the synthesis of reduced glutathione and methionine can scavenge for reactive oxygen species (ROS) such as hydrogen peroxide and hydroxyl radical (Metayer et al., 2008). This may be responsible for the increase in the superoxide dismutase, glutathione peroxidase, and catalase in this study. Generally, the significant effect of the CPM $\times$ MET $\times$ ENZ suggests that the three main effects are interdependent on each other in the determination of the activities of the three antioxidant enzymes studied

\section{Conclusion}

The study elucidated the various effects of cassava peel meal when supplemented with methionine and multienzyme on growing rabbits. This study suggested that cassava peel meal could be included in growing rabbits' diet at $350-\mathrm{g} / \mathrm{kg}$ level but such diets should be supplemented with methionine at $32.53 \%$ higher than the requirement and $5.5-\mathrm{g} / \mathrm{kg}$ multienzyme without deleterious effects on the amino acid digestibility, blood, serum, and antioxidant indices. Thus, the nutritive potential of CPM, which is often limited by cyanide toxicity, can be explored for rabbit feeding if methionine couple with appropriate $5.5 \mathrm{~g} / \mathrm{kg}$ enzyme supplementation level is adopted.

\section{Abbreviations}

ANOVA: Analysis of variance; CF: Crude fiber; CP: Crude protein; CPM: Cassava peel meal; MET: Methionine; ENZ: Multienzyme; RBC: Red blood cell; AST: Aspertate aminotransferase; ALT: Alanine aminotransferase; ALB: Albumin; CRE: Creatinine; CHO: Cholesterol; BIL: Bilirubin; URE: Urea; KEDTA: Potassium ethylene diamine tetraacetic acid; AOAC: Association of Analytical Chemists; GPx: Glutathione peroxidase; SOD: Superoxide dismutase; TPR: Total protein; GLO: Globulin; BDG: Brewer's dried grain; NDF: Neutral detergent fiber; ADF: Acid detergent fiber; ADL: Acid detergent lignin; HCN: Hydrogen cyanide; Leu: Leucine; Lys: Lysine; Iso: Isoleucine; Phe: Phenylalanine; Val: Valine; Met: Methionine; His: Histidine; Thr: Threonine; Cyst: Cystein; PCV: Packed cell volume; HB: Hemoglobin concentration; MCH: Mean cell hemoglobin; MCV: Mean cell volume; MCHC: Mean cell hemoglobin concentration; WBC: White blood cells; LYM: Lymphocytes; PLA: Platelets

\section{Acknowledgements}

The author appreciates Dr. Jimoh O.A, Mr. Ayodele S.O, and Mrs. Oloruntola D.A for their technical supports.

\section{Author's contributions}

ODO designed and managed all activities of the experiment. The author searched and gathered referenced materials and the manuscript. The author reviewed and approved the final manuscript.

\section{Funding}

This research was funded by the Federal Government of Nigeria Tetfund grant 2014

\section{Availability of data and materials}

The dataset generated and analyzed during the current study is available from the corresponding author on a reasonable request.

\section{Competing interest}

The author declares that she have no competing interests.

\section{Ethics approval and consent to participate}

The approval of protocol for this feeding trial was given by the Research Ethics Committee of Agricultural Technology Department, The Federal Polytechnic, Ado Ekiti, Nigeria. The experimental animals were managed following the recommendations and guidelines for applied nutrition experiments in rabbits.

\section{Consent for publication}

Not applicable

Received: 29 November 2019 Accepted: 27 May 2020

Published online: 08 June 2020

\section{References}

Adegbola, T. A., \& Okonkwo, J. C. (2002). Nutritive digestibility and growth rate of rabbits fed varying levels of cassava leaf meal. Nigerian Journal of Animal Production., 29(1), 21-26.

Aebi, H. (1974). Catalase estimation In: Bergmeyer HV (ed.). Methods of enzymatic analysis. Verlag Chemic, New York, 673-84.

Akanmu, M.A., \& Adeyemo, G.O. (2012). The effect of neem and pawpaw leaves supplementation on blood profile of broilers. International Journal of Current Research., 4(12), 272-276.

Aniebo, A. O. (2012). Appraisal of palm oil and methionine as detoxifying agents of residual cyanide in cassava-based broiler starter diets. International Journal of Food, Agriculture and Veterinary Science., 2(1), 153-161 http://www.cibtech. org/jfav.htm.

AOAC (2000). Official method of Analysis. Washington, DC: AOAC International.

Aro, S. O. (2008). Improvement in the nutritive quality of cassava and its byproducts through microbial fermentation. Journal of Biotechnology., 7(25), 4789-4797 https://www.ajol.info/index.php/ajb/article/view/59672.

Ayodele, S. O., Oloruntola, O. D., \& Agbede, J. O. (2016). Effect of Alchornea cordifolia leaf meal inclusion and enzyme supplementation on performance and digestibility of rabbits. World Rabbit Science., 24, 201-206.

Benitez, L.V. (1989). Amino acid and fatty acid profiles in aquaculture nutrition studies, p. 23- 35. In: S.S. De Silva (ed.) Fish Nutrition Research in Asia. In Proc: 
3rd Asian Fish Nutrition Network Meeting. Asian fish. Society Special Publication. Asian Fisheries Society, Manila Philippines.4, 166 p.

Blas, E., Falcao, O., Gidenne, T., Scapinello, C., Pinheiro, V., Garcia, A. I., \& Carabano, R. (2003). Interlaboratory study on ileal digestibility in rabbits: The effect of digesta collection time and a simplification of the procedure. World Rabbit Science., 11, 101-111 https://riunet.upv.es/bitstream/handle/10251/9955/501931-1-SM.pdf

Broom, M.M., Johnson, K.G. (1993). Stress and animal welfare. London Chapman and Hall.

Burnett, N., Mathura, K., Mativier, K. S., Holder, R. B., Brown, G., \& Campbell, M. (2003). An investigation into haematological and serum chemistry parameters of rabbits in Trinidad. World Rabbit Science., 14, 175-185 https:// riunet.upv.es/bitstream/handle/10251/9617/556-1041-1-SM.pdf.

Dennis, M. M. (2005). Clinical textbook for veterinary technicians, (5th ed., ). The USA: Saunders.

FAOStat.(2013). Food and Agriculture Organization of the United Nations.

Freitas, D. M., Vieira, S. L., Angel, C. R., Favero, A., \& Maiorka, A. (2011). Performance and nutrient utilization of broilers fed diets supplemented with a novel mono-component protease. Journal of Applied Poultry Research., 20, 322-334.

Garcia, M., \& Dale, N. (1999). Cassava root meal for poultry. Journal of Applied Poultry Science., 8, 132-137.

Gary, E. I., \& Gary, A. R. (2015). Toxicology of cyanides and cyanogens: Experimental, applied, and clinical aspects. John Wiley and Sons. Pp., 54-80.

Giorgia, M., Mario, G., \& Gilberto, F. (2017). Evaluation of physiological parameters of the plasma oxidative status in rabbits. Journal of Applied Animal Research., 45(1), 315-319.

Goering, H. K., \& Van Soest, P. J. (1970). Forage fiber analysis (apparatus reagents, procedures, and some applications). United States Department of Agriculture, Washington DC: Agriculture Handbook.

Hossain, M. A., Iji, A. A., \& Islam, A. M. F. (2016). Gross responses and ileal digestibility of amino acids and minerals in broiler chickens fed vegetablebased starter diets supplemented with microbial enzymes. Turkish Journal of Veterinary and Animal Science., 40, 1-7. https://doi.org/10.3906/vet-1504-65.

Iyayi, E. A., \& Odueso, O. M. (2003). Response of some metabolic and biochemical indices in rabbits fed varying levels of dietary cyanide. African Journal of Biomedical Research., 6(1), 43-47 http://www.bioline.org.br/pdf?md03008.

Job TA. (1975). Utilization and protein supplementation of cassava for animal feeding and the effect of sulphur sources on cyanide detoxification. Ph.D. thesis. Ibadan, Nigeria: University of Ibadan.

Khadijah, A., Ati, A., Mohammed, S., Saad, A. M., \& Mohammed, H. E. (2009). Response of broiler chicks to dietary monosodium glutamate. Pakistan Veterinary Journal., 29(4), 165-168 http://www.pvj.com.pk/pdf-files/2 9_4/165-168.pdf.

Kiarie, E., Romero, L. F., \& Nyachoti, C. M. (2013). The role of added feed enzymes on promoting gut health in swine and poultry. Nutr. Res. Rev., 26, 71-88 https://doi.org/10.1017/S0954422413000048.

Latimer, K. S., \& Mahaffey, E. A. (2003). Duncan and Prasse's veterinary laboratory medicine: Clinical pathology, (4th ed., pp. 162-192). IA: Iowa State Press. Ames.

Lording, P. M., \& Friend S.C.E. (1991). Interpretation of laboratory results. Australian Veterinary Practitioner., 21(4), 188-193. https://doi.org/10.1111/j.1751-0813. 1971.tb15498.x

Lukuyu, B., Okike, I., Duncan, A., Beveridge, M., \& Blümmel, M. (2014). Use of cassava in livestock and aquaculture feeding programs. ILRI Discussion Paper 25. Nairobi, Kenya: International Livestock Research Institute. Pp. vi, 4-7, 23.

Metayer, S., Seiliez, I., Collin, A., Duchene, S., Mercier, Y., Geraert, P. A., \& Tesseraud, S. (2008). Mechanisms through which sulfur amino acids control protein metabolism and oxidative status. Journal of Nutrition and Biochemistry., 19, 207-215.

Misra, H. P., \& Fridovich, I. (1972). The univalent reduction of oxygen by flavins and quinines. Journal of Biological Chemistry, 247, 188-192.

Morgan, N. K., \& Choct, M. (2016). Cassava: Nutrient composition and nutritive value in poultry diets. Animal Nutrition., 2, 253-261.

Nakajima T. (2015). Roles of sulfur metabolism and rhodanese in detoxification and anti-oxidative stress functions in the liver: Responses to radiation exposure. Medical Science. Monitor, 21,1721-1725. doi: 10.12659/MSM.893234.

Oboh, G., Akindahunsi, A. A., \& Oshodi, A. A. (2002). Nutrient and anti-nutrient content of Aspergillus niger fermented cassava products (flour and gari). Journal of Food Composition andAnalysis, 15, 617-622.

Ogunsipe, M. H., Adejumo, J. O., Agbede, J. O., \& Asaniyan, E. K. (2015). Effect of roxazyme $\mathrm{G} 2 \mathrm{G}$ supplementation on cassava plant meal fed to broiler chickens. Livestock Research for Rural Development, 27(12) http://www.Irrd.org/ Irrd27/12/ogun27240.htm.

Okorie, E. N., \& Ophori, E. A. (2013). Effect of nutritional supplement on the CD4 T-lymphocyte counts of treatment naïve asymptomatic HIV patients in Benin City, Nigeria. Advanced Bioresearch., 4(2), 106-108 https://www. semanticscholar.org/paper/Effect-of-Nutritional-Supplement-on-the-CD4Counts-Ophori/a50cd610f60a7dade9db0fe53c5184f7c8499518.

Oladunjoye, I. O., Ojebiyi, O. O., \& Rafiu, T. A. (2014). High methionine supplementation improves the nutritional value of cassava peel meal for broiler chicken. Livestock Research for Rural Development, 26(4) http://www. Irrd.org//rrd26/4/olad26063.htm.

Oloruntola, O. D. (2018). Influence of enzyme supplementation on rabbits fed rumen liquor with poultry waste fermented cassava peels based diets. Animal Research International., 15(1), 2950-2964 https://www.ajol.info/index. php/ari/article/download/186957/176230.

Oloruntola, O.D., Agbede, J.O., Onibi,G.E., \& Igbasan, F.A.,(2016). Replacement value of rumen liquor fermented cassava peels for maize in growing rabbit diets. Archivos de Zootecnia. 65 (249), 89-97.

Oloruntola, O.D., Agbede, J.O., Onibi., G.E., Igbasan, F.A., Ogunsipe, M.H., \&Ayodele, S.O. (2018). Rabbits fed fermented cassava starch residue II: Enzyme supplementation influence on performance and health status. Archivos de Zootecnia. 67 (260), 588-595.

Oloruntola, O.D., Agbede, J.O., Onibi, G.E., \& Igbasan, F.A., (2015). Composition of cassava (Manihot spp.) peels fermented with bovine rumen liquor and different nitrogen sources. Journal of Global Agriculture and Ecology,2(1), 2635.http://ikprress.org/index.php/JOGAE/article/view/278

Oloruntola, O. D., Ayodele, S. O., Agbede, J. O., \& Oloruntola, D. A. (2016). Effect of feeding broiler chicken with diets containing Alchornea cordifolia leaf meal and enzyme supplementation. Archivos de Zootecnia., 65(252), 489-498.

Oloruntola, O. D., Ayodele, S. O., Agbede, J. O., Oloruntola, D. A., Ogunsipe, M. H., \& Omoniyi, I. S. (2016). Effect of Alchorneal cordifolia leaf meal and enzyme supplementation on growth, haematological, immunostimulatory and serum biochemical response of rabbits. Asian Journal of Biological and Life Sciences., 5(2), 190-195.

Oloruntola O.D., Ayodele S.O., Jimoh A.O., \&Agbede J.O (2019). Dietary cassava peel meal, methionine, and multi-enzyme supplementation in rabbits' nutrition: effect on growth, digestibility, and carcass traits. Journal of Basic and Applied Zoology. 80:46. DOI : 10.1186/s41936-019-0117-z.

Onifade, A. A., \& Tewe, O. O. (1993). Alternative tropical energy feed resources in rabbit diets: Growth performance, diet's digestibility, and blood composition. World Rabbit Science, 1(1), 17-24.

Prathibha, S., Nambisan, B., \& Leelarnma, S. (1995). Enzyme inhibitors in tuber crops and their thermal stability. Plant Foods for Human Nutrition, 48, 247257.

Rotruck, J. T., Pope, A. L., Ganther, H. E., Hafeman, D. G., \& Hoekstra, W. G. (1973). Selenium: Biochemical role as a component of glutathione peroxidase. Science., 179, 588-590.

Shou Y., Gunasekar P.G., Borowitz J.L \& Isom G.E (2000). Cyanide-induced apoptosis involves oxidative stress-activated NFKB in cortical neurons. Toxicology and Applied Pharmacology, 164, 196-205.

Surai, P. F. (2002). Selenium in poultry nutrition 2. Reproduction, egg and meat quality and practical application. World's Poultry Science, 58, 431-450 https:// doi.org/10.1079/WPS20020032.

Wrobel, M., Jurkowska, H., Sliwa, L., \& Srebro, Z. (2004). Sulfurtransferases and cyanide detoxification in mouse liver, kidney, and brain. Toxicology Mechanism and Methods., 14(6), 331-337.

\section{Publisher's Note}

Springer Nature remains neutral with regard to jurisdictional claims in published maps and institutional affiliations. 